\title{
ANÁLISIS DE FALLA DE UN TALUD EN UN SECTOR URBANO DEL MUNICIPIO DE OCAÑA, COLOMBIA
}

\section{FAILURE ANALYSIS OF A SLOPE IN AN URBAN SECTOR OF THE MUNICIPALITY OF OCAÑA, COLOMBIA}

\author{
MSc. Romel Jesús Gallardo Amaya* \\ *Universidad Francisco de Paula Santander Ocaña, Facultad de Ingenierías, Grupo de \\ Investigación en Construcción, Geotecnia y Medio Ambiente-GIGMA. \\ Vía Acolsure, Sede algodonal, Ocaña, Norte de Santander, Colombia. \\ Tel.: +57 5690088 Ext. 509. \\ E-mail: rjgallardoa@ufpso.edu.co.
}

\begin{abstract}
Resumen: El barrio San Fermín se ubica en el sector sur de la ciudad de Ocaña, en una zona de ladera con una alta intervención antrópica. Esto ha potenciado que se presentan problemas de erosión y de estabilidad de taludes, que mantienen en un nivel de riesgo considerable a una parte de sus habitantes. La investigación buscó analizar las condiciones que conllevaron a la falla de un talud en una de las terrazas que conforman el barrio y establecer las condiciones de estabilidad del talud posteriores a la falla, de modo que se pueda conocer el nivel de amenaza asociado. Se realizó una detallada investigación de campo que permitió establecer el modelo geotécnico más adecuado para el análisis. Los resultados permitieron definir las condiciones en las que se presentó la falla del talud y establecer, que posterior al deslizamiento, el talud continúa en condiciones muy susceptibles a fallar nuevamente.
\end{abstract}

Palabras clave: Análisis de estabilidad, deslizamiento, factor de seguridad, retroanálisis, sensibilidad.

Abstract: The San Fermín neighborhood is located in the southern sector of the city of Ocaña, in a hillside zone with high anthropic intervention. This has enhanced the problems of erosion and slope stability, which maintain a considerable level of risk to a part of its inhabitants. The investigation sought to analyze the conditions that led to the failure of a slope on one of the terraces that make up the neighborhood and establish the conditions of stability of the slope after the fault, so that the level of associated threat can be known. A detailed field investigation was carried out that allowed establishing the most appropriate geotechnical model for the analysis. The results allowed to define the conditions in which the slope failure occurred and to establish, that after the landslide, the slope continues in conditions very susceptible to fail again.

Keywords: Stability analysis, landslide, safety factor, backanalysis, sensitivity. 


\section{INTRODUCCIÓN}

Aunque los deslizamientos de tierra se consideran como fenómenos naturales de origen geológico, su materialización, en muchos casos, se produce por la intervención humana (OEA, 1993; Cuanalo et al., 2011). Un deslizamiento de tierra, o movimiento en masa (Cruden, 1991), consiste en un movimiento hacia abajo o hacia afuera, por efecto de la gravedad, de materiales que pueden ser bloques o fragmentos de roca, suelo o depósitos de origen antrópico (SGC, 2017; Suárez, 2009), y su ocurrencia son una de las mayores causas de desastres en todo el mundo, con las consecuentes pérdidas socio-económicas y ambientales (Arias, 2012); (R García et al., 2018).

Para que en un talud o una ladera se presente un deslizamiento, es necesario que su condición de estabilidad o "inestabilidad" se vea influenciada por la interacción entre una serie de factores que se clasifican en condicionantes y desencadenantes o como los definió Terzaghi (1950) como causas internas y externas; siendo los factores relacionados a las condiciones del suelo, los considerados como los determinadores de los deslizamientos (Krynine \& Judd, 1980), siendo estos factores afectados de manera progresiva, o incluso inmediata, por los factores desencadenantes o contribuyentes (Montero, 1991; Popescu, 2002; Cuanalo \& Oliva, 2011).

En todo talud siempre está presente la interacción entre unas fuerzas que propenden por el movimiento descendente del material que lo conforma y otras que se oponen a dicho movimiento a lo largo de una superficie de falla, la relación entre estas fuerzas determina el factor de seguridad (Yang et al., 2016; Oliva et al., 2018)), cuya definición, en Ingeniería Geotécnica, se establece en el Reglamento Colombiano de Construcción Sismo Resistente (2010) como "la relación entre el esfuerzo cortante último resistente o esfuerzo cortante a la falla y el esfuerzo cortante actuante".

El Factor de Seguridad de un talud se determina mediante modelación matemática con el objeto de analizar sus condiciones de estabilidad (Gallardo et al., 2013; Ucar y Belandría, 2015; Oliva et al., 2019). La investigación presenta los resultados de análisis de las condiciones de un talud ubicado en un sector urbano de la ciudad de Ocaña, Colombia, estableciendo el factor de seguridad antes y después de la ocurrencia del deslizamiento, para lo cual se realizó la modelación mediante un software basado en la teoría de equilibrio límite y para los parámetros físico-mecánicos obtenidos mediante diferentes métodos (Suárez et al., 2016; Guerra et al., 2016; Mesa y Tejeda, 2019).

\section{MATERIALES Y MÉTODOS}

\subsection{Características del talud}

\subsubsection{Localización}

El barrio San Fermín se ubica en el sector sur de la ciudad de Ocaña Norte de Santander y, la zona de estudio se localiza en las coordenadas 1'401.532 N y $1^{\prime} 078.905$ E $\left(8^{\circ} 13^{\prime} 35.05^{\prime \prime} \mathrm{N}, 73^{\circ} 21^{\prime} 40.77^{\prime \prime W}\right)$ y posee una extensión de $1.35 \mathrm{Ha}$. En la figura 1 se puede apreciar la distribución general de este asentamiento urbano.

\subsubsection{Antecedentes y problemática actual}

El barrió San Fermín es un asentamiento urbano de la ciudad producto de invasiones entre los años 1995 y 1996, y en la actualidad la mayoría de sus predios no están legalizados. La construcción de viviendas en esta zona se ha realizado sin una correcta planeación y sin ningún tipo de control, realizando cortes inadecuados a la ladera.

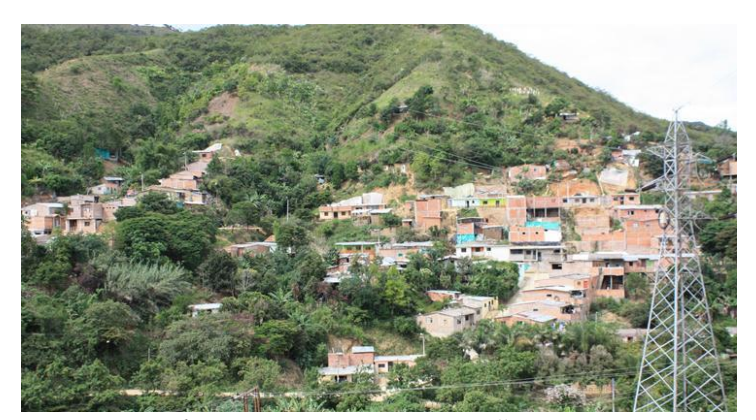

Fig. 1. Panorámica Barrio San Fermín

Fuente: Autor.

La construcción de nuevas viviendas y el mejoramiento de las existentes ha incrementado el área de terrazas y por consiguiente los cortes a la ladera, razón que ha potenciado los deslizamientos sucesivos en época invernal. En la figura 2 se puede apreciar como desde 2009 a 2014 el talud en la terraza media avanzó $4.2 \mathrm{~m}$ en el sector izquierdo, $5.4 \mathrm{~m}$ en el sector derecho y $9.6 \mathrm{~m}$ en el sector central. 


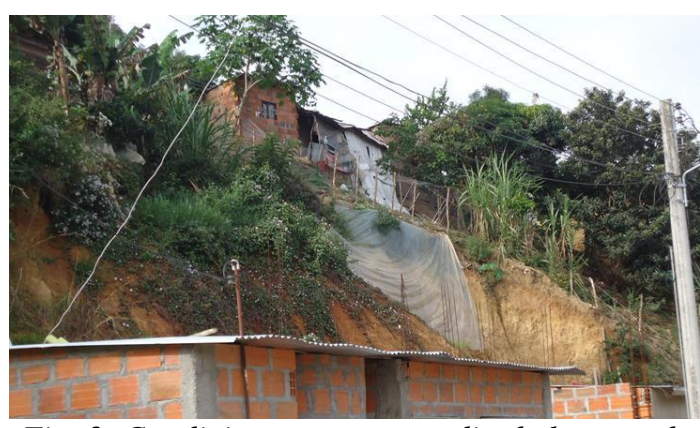

Fig. 2. Condiciones terraza media de la zona de estudio. Fuente: Autor

Además, a partir de reconocimiento y visitas de campo se identificó que el sector correspondiente a la terraza media, en la cual se encuentran los taludes más críticos, se ve afectado considerablemente por la constante infiltración de agua proveniente de una tubería de concreto de 16 ", que corresponde a la línea de aducción de una de las plantas de tratamiento de la ciudad, la cual se ubica a $30 \mathrm{~m}$ arriba de los escarpes de los taludes críticos del sector de análisis. Esta situación hace que se presente afloramiento de un caudal importante de agua en varios puntos de la ladera, tal como se muestra en la figura 3.

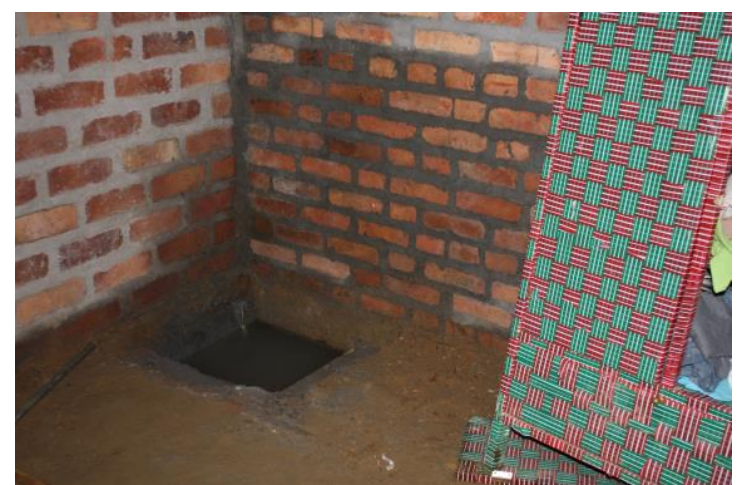

Fig. 3. Afloramiento de agua en la base de la terraza media de la ladera. Fuente: Autor

\subsection{Investigación geotécnica}

El programa de laboratorios y ensayos para la adquisición de información contemplo el desarrollo de sondeos por rotación y percusión, con su respectiva toma de muestras alteradas e inalteradas (Gallardo et al., 2017), las cuales se emplearon para determinar las propiedades físicas y mecánicas de los geomateriales que componen el sector de estudio.

\subsubsection{Sondeos geotécnicos}

En total se realizaron 5 sondeos con profundidades variables entre $2.65 \mathrm{~m}$ y $11.65 \mathrm{~m}$. Dos corresponden a sondeos continuos con un equipo de rotación y los tres restantes a sondeos de percusión realizando ensayos de penetración estándar - SPT. En la figura 4 se puede apreciar la ubicación de cada sondeo, representados por los círculos en rojo.

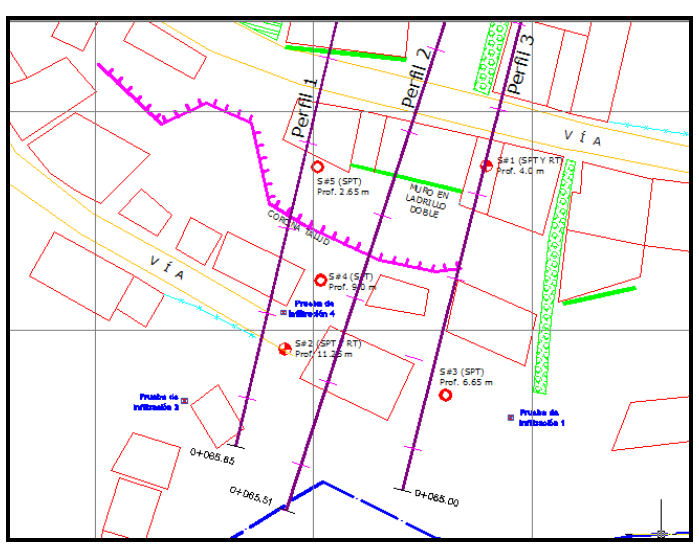

Fig. 4. Localización de sondeos. Fuente: Autor

\subsection{Modelo geotécnico}

Para el análisis de estabilidad se integró la información geológica, topográfica, información de la inspección visual, resultados de los sondeos y ensayos de laboratorio, lo que permitió establecer el modelo geotécnico para el análisis de uno de los taludes más críticos identificados en la zona, que corresponde al perfil 1 (Sección izquierda) de la figura 4, para el cual su geometría y estratigrafía corresponde a un perfil compuesto por suelos residuales entre $0.0 \mathrm{~m}$ y $7.0 \mathrm{~m}$ en la parte superior del talud y entre $0.0 \mathrm{~m}$ y $2.5 \mathrm{~m}$ en la parte baja del mismo. Por debajo de estos materiales se encuentra la continuación de horizontes de materiales del saprolito y transición del saprolito a roca parcialmente meteorizada (Monroy et al., 2014).

Con respecto a las propiedades de los geomateriales que integran el modelo geotécnico se utilizó el sistema de parámetros efectivos de resistencia, de acuerdo a la teoría de MohrCoulomb obtenidos en ensayos de corte directo drenado, en correlaciones con resultados de ensayos SPT, en estudios anteriores realizados en la zona de estudio y los obtenidos por análisis de 
sensibilidad y retroanálisis de los deslizamientos ocurridos en la zona de estudio, en la tabla 1 se muestran los parámetros utilizados para el modelo geotécnico.

Para el análisis de estabilidad con obras proyectadas se realizó el análisis tomando para el suelo residual medio duro (horizonte donde se pueden desarrollar las potenciales superficies de falla de acuerdo a lo observado en visita de campo) los valores encontrados mediante el "retro cálculo" (ASCE, 1999). En la Tabla 2 se detalla los resultados del análisis que posteriormente se explican en detalle.

\section{Tabla 1: Parámetros utilizados en el modelo}

\begin{tabular}{|c|c|c|c|c|}
\hline \multicolumn{2}{|r|}{ Material } & $\begin{array}{c}\text { Peso } \\
\text { unitario } \\
\mathbf{k N} / \mathbf{m}^{3}\end{array}$ & $\begin{array}{c}\text { Ángulo } \\
\text { de } \\
\text { fricción } \\
\text { " } \phi "\left({ }^{\circ}\right)\end{array}$ & $\begin{array}{c}\text { Cohesión } \\
\text { kN/m² }\end{array}$ \\
\hline \multirow{5}{*}{ 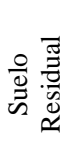 } & Muy Suelto & 18.44 & 21.77 & 17.60 \\
\hline & Suelto & 18.93 & 21.77 & 17.60 \\
\hline & Medio Duro & 19.82 & 23.17 & 26.64 \\
\hline & Duro & 20.31 & 26.17 & 20.52 \\
\hline & Saprolito & 20.99 & 27.71 & 27.76 \\
\hline
\end{tabular}

Fuente: Autor.

\section{Tabla 2: Parámetros obtenidos con retro cálculo} para falla del talud analizado (perfil 1)

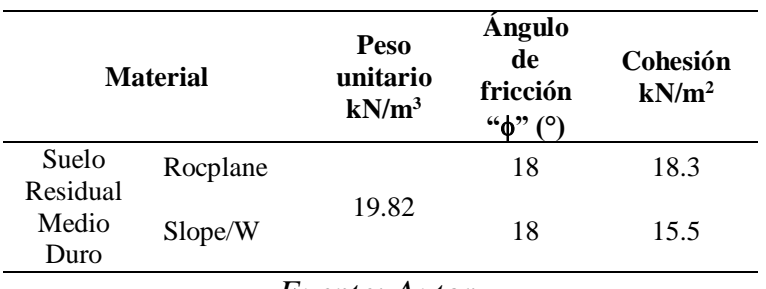

Fuente: Autor.

El modelo geotécnico realizado se analizó con el uso del software Slope $/ \mathrm{W}^{\circledR}$. Dicho análisis se realizó antes y después del deslizamiento ocurrido en el talud, en las figuras 5 y 6 se puede apreciar el correspondiente modelo geotécnico para la condición antes del deslizamiento y para la condición actual

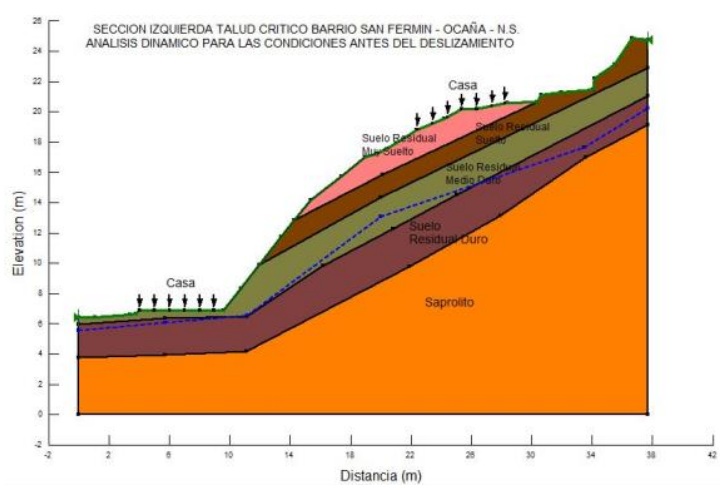

Fig. 5. Modelo geotécnico para el talud antes del deslizamiento. Fuente: Autor.

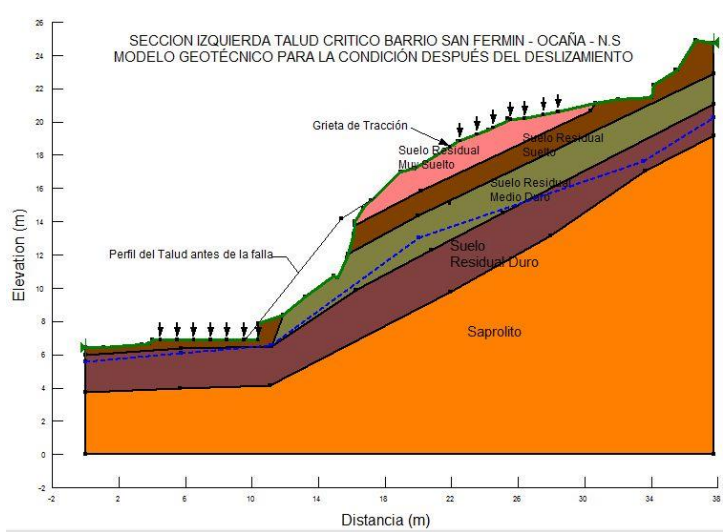

Fig. 6. Modelo geotécnico para el talud después del deslizamiento. Fuente: Autor.

\section{RESULTADOS Y ANÁLISIS}

\subsection{Análisis de sensibilidad y retro cálculo}

Se realizó el análisis de sensibilidad y retro cálculo para el talud en condición más crítica en la zona de estudio (perfil 1), antes de que se presentara la falla cuyo escarpe se evidencia en la actualidad, y que corresponde a un deslizamiento planar de un bloque completo de material que incluye horizontes del perfil de meteorización presente en el lugar. Teniendo en cuenta lo observado en campo este deslizamiento tiene la superficie de falla localizada principalmente en el horizonte de suelos medio duros, por lo cual en el análisis de sensibilidad se han variado las propiedades de cohesión y fricción para este material.

Los resultados del análisis de sensibilidad mostrados en la tabla 3 se graficaron en la figura 7 , buscando la condición para la cual se presenta un factor de seguridad, en la situación antes del deslizamiento, igual a 1.0. 


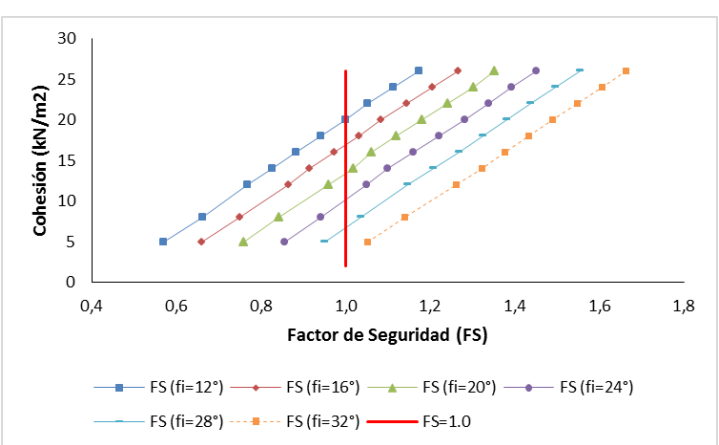

Fig. 7. Resultados del análisis de sensibilidad y del retro cálculo para el talud analizado.

Fuente: Autor.

Tabla 3: Resumen del análisis de sensibilidady retro cálculo para el talud analizado

\begin{tabular}{ccccccc}
\hline $\begin{array}{c}\mathbf{C} \\
\mathbf{k P a}\end{array}$ & $\begin{array}{c}\mathbf{F S} \\
\boldsymbol{\phi}=\mathbf{1 2}\end{array}$ & $\begin{array}{c}\mathbf{F S} \\
\boldsymbol{\phi}=\mathbf{1 6}\end{array}$ & $\begin{array}{c}\mathbf{F S} \\
\boldsymbol{\phi}=\mathbf{2 0}\end{array}$ & $\begin{array}{c}\mathbf{F S} \\
\boldsymbol{\phi}=\mathbf{2 4}\end{array}$ & $\begin{array}{c}\mathbf{F S} \\
(\boldsymbol{\phi}=\mathbf{2 8})\end{array}$ & $\begin{array}{c}\mathbf{F S} \\
(\boldsymbol{\phi}=\mathbf{3 2})\end{array}$ \\
\hline 5 & 0,570 & 0,660 & 0,759 & 0,856 & 0,951 & 1,053 \\
8 & 0,663 & 0,751 & 0,843 & 0,941 & 1,036 & 1,141 \\
12 & 0,769 & 0,866 & 0,959 & 1,049 & 1,146 & 1,262 \\
14 & 0,826 & 0,915 & 1,018 & 1,100 & 1,207 & 1,322 \\
16 & 0,884 & 0,973 & 1,062 & 1,160 & 1,268 & 1,377 \\
18 & 0,942 & 1,031 & 1,120 & 1,221 & 1,326 & 1,433 \\
20 & 1,000 & 1,084 & 1,181 & 1,282 & 1,381 & 1,490 \\
22 & 1,052 & 1,145 & 1,241 & 1,339 & 1,438 & 1,548 \\
24 & 1,113 & 1,206 & 1,302 & 1,393 & 1,496 & 1,606 \\
26 & 1,174 & 1,267 & 1,353 & 1,451 & 1,554 & 1,663 \\
\hline
\end{tabular}

Fuente: Autor.

\subsection{Cálculo del factor de seguridad}

El cálculo de los factores de seguridad se realiza a partir del análisis de estabilidad, para conocer el valor que presenta el talud actualmente y el valor que tenían antes de ocurrir el deslizamiento. El análisis se realiza para falla en bloque con el software Slope $/ \mathrm{W}^{\circledR}$, el cual emplea la teoría del equilibrio límite para analizar los factores de seguridad en deslizamiento de taludes, incorporando las metodologías de Bishop, Fellenius, Jambu y Spencer.

\subsubsection{Antes del deslizamiento}

El análisis se realiza para la condición estática y dinámica, y en esta última se consideró que $\mathrm{K}_{\mathrm{ST}} / \mathrm{a}_{\max }$ de 0.8, de acuerdo a la Tabla H.5.2-1 de la NSR-10 (Suelos, enrocados y macizos rocosos muy fracturados (RQD<50\%) (Terzaghi, et al., 1967). Este valor también podría tomarse hasta de Universidad de Pamplona

I. I. D. T. A.
0.5 teniendo en cuenta que la fuerza sísmica no es una fuerza que actúa todo el tiempo.

En la figura 8 se aprecia el resultado para el talud analizado antes del deslizamiento en su condición dinámica con los parámetros de resistencia obtenidos con los ensayos de laboratorio.

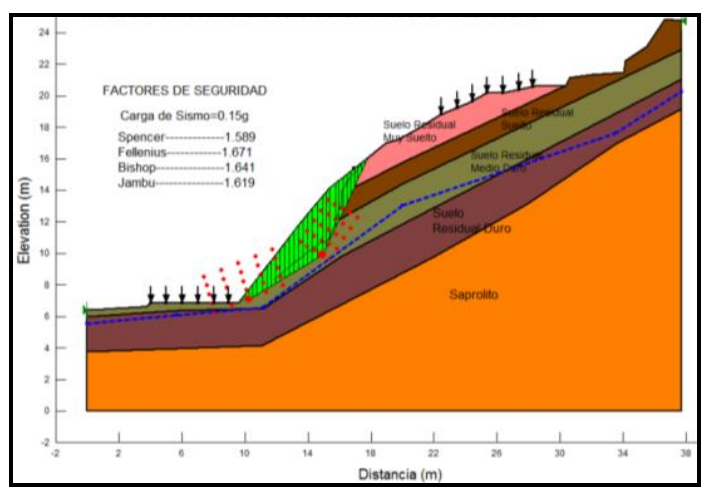

Fig. 8. Análisis del talud antes del deslizamiento, condición dinámica. Parámetros de resistencia obtenidos con ensayos de laboratorio.

Fuente: Autor.

Así mismo, en la figura 9 se aprecia el resultado para el talud analizado con los parámetros de resistencia obtenidos con el retro cálculo.

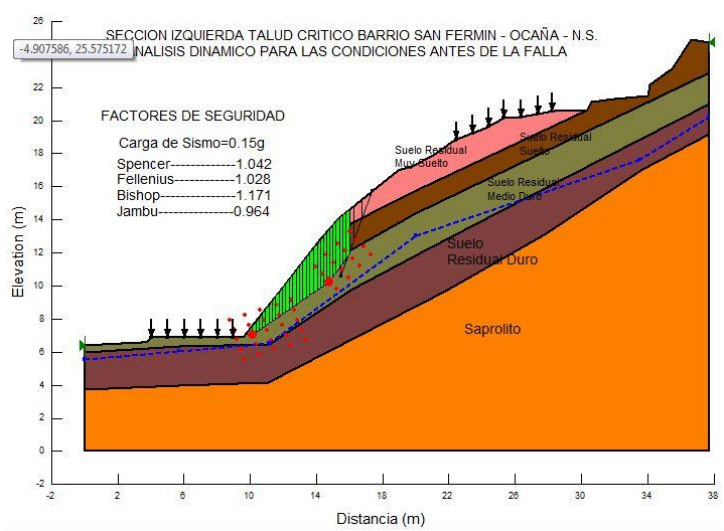

Fig. 9. Análisis del talud antes del deslizamiento, condición dinámica. Parámetros de resistencia obtenidos con retro cálculo. Fuente: Autor.

\subsubsection{Despues del deslizamiento}

De igual forma se hizo el respectivo análisis para el talud después del deslizamiento, en las figuras $10 \mathrm{y}$ 11 se aprecian los resultados obtenidos para la condición dinámica, con los parámetros resistencia obtenidos en laboratorio y con retro calculo respectivamente. 


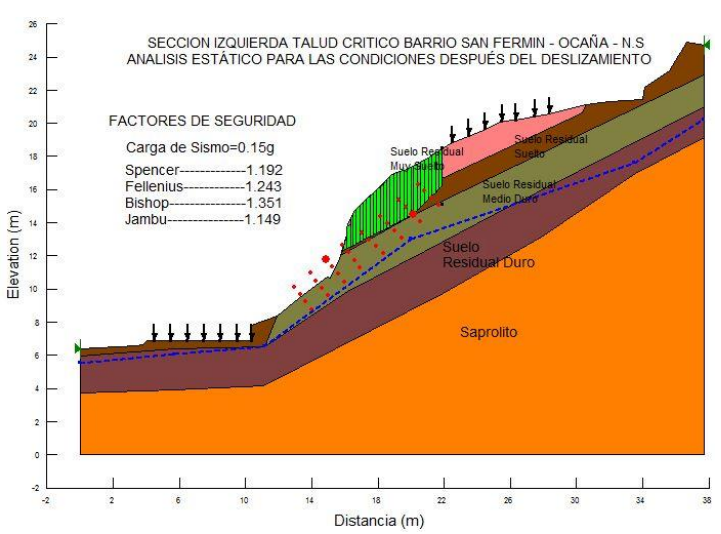

Fig. 10. Análisis del talud después del deslizamiento, condición dinámica. Parámetros de resistencia obtenidos con ensayos de laboratorio.

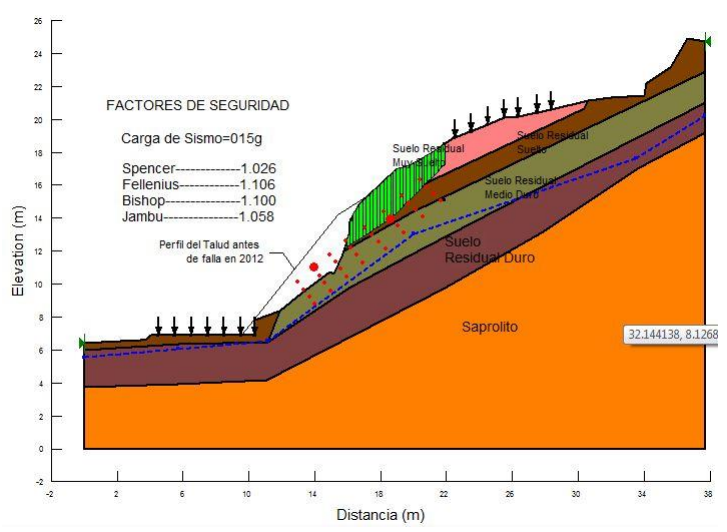

Fig. 11. Análisis del talud después del deslizamiento, condición dinámica. Parámetros de resistencia obtenidos con retro cálculo.

Los resultados obtenidos con el análisis del talud a través de Slope $/ \mathrm{W}^{\circledR}$ tanto en condición estática como dinámica, antes y después del deslizamiento, y para cada conjunto de parámetros de resistencia obtenidos mediante diferentes procedimientos, se resumen en la tabla 4.

\section{Tabla 4: Resumen del análisis de estabilidad para el talud}

\begin{tabular}{|c|c|c|c|}
\hline \multirow{2}{*}{ Talud/condición } & \multirow{2}{*}{$\begin{array}{c}\begin{array}{c}\text { Parámetros } \\
\text { de }\end{array} \\
\text { Resistencia } \\
\end{array}$} & \multicolumn{2}{|c|}{ Factor de Seguridad } \\
\hline & & Estático & Dinámico \\
\hline \multirow{3}{*}{$\begin{array}{l}\text { Talud izquierdo } \\
\text { (perfil 1) / antes del } \\
\text { deslizamiento }\end{array}$} & $\begin{array}{l}\text { Datos corte } \\
\text { Directo: } \\
\phi=23.17^{\circ} \\
\mathrm{c}=26.6 \mathrm{kPa}\end{array}$ & 1.906 & 1.589 \\
\hline & $\begin{array}{l}\text { Datos } \\
\text { RocLab: } \\
\phi=11.85^{\circ} \mathrm{c}=6 \\
\mathrm{kPa}\end{array}$ & 0.609 & 0.494 \\
\hline & $\begin{array}{l}\text { Datos Retro } \\
\text { cálculo: } \phi=18^{\circ} \\
\mathrm{c}=18.3 \mathrm{kPa}\end{array}$ & 1.243 & 1.042 \\
\hline
\end{tabular}

Universidad de Pamplona I. I. D. T. A.

\begin{tabular}{clll}
\hline & Datos corte & & \\
& $\begin{array}{l}\text { Directo: } \\
\phi=23.17^{\circ}\end{array}$ & 1.463 & 1.192 \\
& $\begin{array}{l} \\
\mathrm{c}=26.6 \mathrm{kPa}\end{array}$ & & \\
\cline { 2 - 3 } $\begin{array}{c}\text { Talud izquierdo } \\
\text { (perfil 1) / después }\end{array}$ & $\begin{array}{l}\text { Datos } \\
\text { RocLab: }\end{array}$ & & \\
del deslizamiento & $\phi=11.85^{\circ} \mathrm{c}=6$ & 0.716 & 0.565 \\
& $\mathrm{kPa}$ & & \\
\cline { 2 - 4 } & Datos Retro & & \\
& $\begin{array}{l}\text { Cálculo } \phi=18^{\circ} \\
\mathrm{c}=18.3 \mathrm{kPa}\end{array}$ & 1.274 & 1.026 \\
& & & \\
\hline
\end{tabular}

De acuerdo a los resultados obtenidos de los factores de seguridad se evidencia que los parámetros con los que mejor se pudo establecer las condiciones de falla del talud, son los que se obtuvieron con ayuda del análisis de sensibilidad y retro cálculo.

\section{CONCLUSIONES}

La aplicación de análisis de sensibilidad y la metodología del retro cálculo son útiles para la determinación de parámetros de resistencia que permitan simular de forma adecuada superficies de falla de un talud, dado que se puede establecer los valores de fricción y cohesión más probables en el momento de ocurrencia de esta. Dichos resultados son relevantes para análisis de estabilidad en las condiciones después de ocurrido un deslizamiento y en especial para el análisis incorporando procesos constructivos de estabilización.

Los análisis realizados al talud en las condiciones antes de la falla permitieron establecer que incluso en condición estática, el talud se encontraba en condición de falla. La superficie de falla encontrada a través del análisis con software basado en equilibrio límite es muy cercana a la evidenciada en campo después del deslizamiento.

Los resultados de factor de seguridad que se obtienen para los parámetros determinados con pruebas de laboratorio, para casos particulares como el de esta investigación, no permiten simular la condición de falla del talud, puesto que incluso para la condición dinámica se obtienen factores de seguridad lejos de la falla. Los resultados obtenidos con parámetros deducidos con información de campo procesada a través del software RocLab, son demasiados bajos para las condiciones reales de falla.

Los factores de seguridad que permitió obtener el anpalisis del talud en las condiciones después de la falla, indican que este se encuentra en una 
condición que favorecerá la ocurrencia de un nuevo deslizamiento, lo cual indica un nivel de amenaza que requiere la implementación de medidas estructurales para la reducción del riesgo latente a que están expuestos los habitantes de este sector de la ciudad.

\section{AGRADECIMIENTOS}

Mis agradecimientos a la División de Investigación y Extensión de la Universidad Francisco de Paula Santander Ocaña, por el apoyo brindado para el desarrollo del proyecto de investigación: Estabilidad de Ladera Urbana en el municipio de Ocaña.

\section{REFERENCIAS}

Arias, T. B. (2012). Deslizamientos en taludes inducidos por altas precipitaciones en vías intermunicipales en Colombia, Universidad Libre, Bogota, D.C., Colombia. https://repository.unilibre.edu.co/bitstream/ha ndle/10901/7116/AriasTorresBenjamin2012.p $\mathrm{df}$ ? sequence $=1 \&$ is Allowed $=\mathrm{y} \quad$ (Consultado: 18 de julio 2019)

Los Angeles Section Geotechnical Group, ASCE. (1999). Recommended procedures for implementation of DMG special publication 117 guidelines for analyzing and mitigating landslide hazards in California. Los Angeles, EEUU. http://www.scec.org/resources/ catalog/ hazardmitigation.html (Consultado: 18 de julio 2019)

Asociación Colombiana de Ingeniería Sísmica, AIS. (2010). Reglamento Colombiano de Construcción Sismo Resistente NSR-10. AIS, Bogotá, Colombia.

Cuanalo, C. O. A. (2011). Inestabilidad de laderas. Deslizamientos y factores desencadenantes: Desastres por Fenómenos Geológicos de Inestabilidad de Laderas. Factores Condicionantes y Desencadenantes, Editorial Académica Española, Alemania.

Cuanalo, C. O. A., Oliva, G. A. O. y Gallardo, A. R. J. (2011). "Inestabilidad de laderas: Influencia de la actividad humana". ELEMENTOS, Vol. 84, No. 1.

Gallardo A., R. J., Cuanalo C., O. A., Quintero L., L., Muñoz, A. A. y Martínez, C. A. (2017). Análisis del comportamiento de suelos de alta plasticidad con la adición del material de residuo en la fabricación de ladrillo cerámico.
REVISTA COLOMBIANA DE TECNOLOGÍAS DE AVANZADA, ISSN: 1692-7257 Vol. 1, No. 29.

Gallardo A., R. J., Guerrero B., T. E. y Macgregor T., A. A. (2013). "Investigación Geotécnica para la estabilización de las laderas del Barrio San Fermín, municipio de Ocaña, departamento de Norte de Santander (Colombia)". Revista INGECUC, Vol. 9, No. 2.

Guerra C., Pinzón J., Prada L. F., y Ramos A. M. (2016). Modelación multiescala de estabilidad de taludes en mezclas de bloques embebidos en matriz fina (BIM). En ISRM 2nd International Specialized Conference on Soft Rocks, Cartagena, Colombia.

Krynine, D.P. y Judd, W. R. (1980). Principles of Engineering Geology and Geotechnics, McGraw Hill, New York, USA.

Mesa L., M. y Tejeda P., E. (2018). “Análisis de la estabilidad de taludes en terraplenes mediante los métodos de equilibrio límite y el método de elementos finitos". Revista Cubana de Ingeniería. Vol. 9, No, 1.

Monroy G., J. E., Cruz H., R. A. y Torres C. M. (2014). Nonlinear model for analysis of soilfoundation interaction. REVISTA COLOMBIANA DE TECNOLOGÍAS DE AVANZADA, ISSN: 1692-7257 Vol. 1, No. 23.

Montero, O. J. (1991). Inventario de deslizamientos en Suramérica. ISSMGE IX Congreso Panamericano de Mecánica de Suelos e Ingeniería de Fundaciones, Viña del Mar, Chile.

Oliva G., A. O., Berumen R., M. A., Gallardo A., R. J. y Jaramillo H. Y. (2019). "Terrain instability in the Tijuana metropolitan area: Analysis of a failure in the access road to a industrial park". Engineering Failure Analysis, Vol. 104.

Oliva G., A. O., Ruiz P., A. F., Gallardo A., R. J. y Jaramillo H. Y. (2018). "Landslide risk assessment in slopes and hillsides. Methodology and application in a real case". DYNA, Vol. 86, No. 208.

Organización de Estados Americanos, OEA. (1993). Manual Sobre el Manejo de Peligros Naturales en la Planificación para el Desarrollo Regional Integrado, Washington. D.C.https://www.oas.org/dsd/publications/uni t/oea65s/begin.htm\#Contents. (Consultado: 15 de enero 2019) 
Popescu M. E. (2002). Landslide causal factors and landslide remediatial options. En 3rd International Conference on Landslides, Slope Stability and Safety of Infrastructures, Singapore.

R García-León, E Flórez, C Acevedo (2018). Caracterización térmica de mezclas de arcillas utilizadas en la fabricación de productos de mampostería para la construcción. Revista Colombiana de Tecnologías de Avanzada, ISSN: 1692-7257

Ramírez, M. y Sánchez E. (2014). ’Manejo del riesgo que generan los taludes de la quebrada La Seca, del municipio de Envigado", Cuaderno ACTIVA, Vol. 1, No. 6.

Servicio Geológico Colombiano, SGC. (2017). Clasificación de movimientos en masa y su distribución en terrenos geológicos de Colombia, Publicaciones Servicio Geológico Colombiano, Bogotá, D.C., Colombia.
Suárez B., L. O. \& Montoya A., E. A. (2016). "Programa en código abierto para el análisis bidimensional de estabilidad de taludes por el método de equilibrio". Revista de la Facultad de Ciencias, Vol. 5, No. 2.

Suárez, D. J. (2009). Deslizamientos, Análisis geotécnico. División de publicaciones UIS, Bucaramanga, Colombia.

Terzaghi, K. (1950). Mechanisms Of Landslides, Application of Geology to Engineering Practice, Geological Society of America, Berkley, USA.

Terzaghi, K. y Peck, R. B. (1967). Soil Mechanics in Engineering Practice. Wiley, New York. USA.

Ucar R. y Belandria N. (2015). “Cálculo del factor de seguridad en la estabilidad de taludes considerando rotura circular". Geominas, Vol. 43, No. 66.

Yang T., Yeung M.R., Yang B., Liu Y. and Yang Y. (2016). "Three-dimensional stability of landslides based on local safety factor". Journal of Mountain Science, Vol. 13, No. 9. 